\title{
Abundance of TRAIL attenuated by HIF2a and c-FLIP affects malignancy in renal cell carcinomas
}

\author{
Takahiro Isono ${ }^{1}$, Tokuhiro Chano ${ }^{2}$, Tetsuya Yoshida ${ }^{3}$, Susumu Kageyama ${ }^{3}$, Akihiro \\ Kawauchi $^{3}$, Junji Yonese ${ }^{4}$ and Takeshi Yuasa ${ }^{4}$ \\ ${ }^{1}$ Central Research Laboratory, Shiga University of Medical Science, Otsu, Shiga 520-2192, Japan \\ ${ }^{2}$ Department of Clinical Laboratory Medicine, Shiga University of Medical Science, Otsu, Shiga 520-2192, Japan \\ ${ }^{3}$ Department of Urology, Shiga University of Medical Science, Otsu, Shiga 520-2192, Japan \\ ${ }^{4}$ Department of Urology, Cancer Institute Hospital, Japanese Foundation for Cancer Research, Tokyo 135-8550, Japan \\ Correspondence to: Takahiro Isono, email: isono@belle.shiga-med.ac.jp \\ Keywords: tumor necrosis factor related apoptosis-induced ligand; hypoxia inducible factor 2-alpha; cellular FLICE (FADD-like IL-1 \\ beta-converting enzyme)-inhibitory protein; renal cell carcinomas; apoptosis \\ Received: November 15, $2017 \quad$ Accepted: April 05, $2018 \quad$ Published: May 01, 2018 \\ Copyright: Isono et al. This is an open-access article distributed under the terms of the Creative Commons Attribution License 3.0 \\ (CC BY 3.0), which permits unrestricted use, distribution, and reproduction in any medium, provided the original author and source \\ are credited.
}

\section{ABSTRACT}

Dormant cancer cells are starvation-resistant leading to problems in the management of cancer. In renal cell carcinomas (RCCs), starvation-resistant cells are resistant to various currently available therapies. However, targeting hypoxia inducible factor 2-alpha (HIF2-alpha) induces cell death in dormantlike/starvation-resistant RCCs. This study showed that the apoptotic cell death caused by tumor necrosis factor (TNF)-related apoptosis-induced ligand (TNFSF10/ TRAIL) was attenuated by CASP8 and FADD-like apoptosis regulator (CFLAR/CFLIP) following HIF2-alpha activation, despite the high expression of TRAIL in such RCCs. Knockdowns of TRAIL averted apoptotic cell death caused by HIF2-alpha inhibition in starvation-resistant RCCs. Knockdowns of both HIF2-alpha and C-FLIP augmented apoptotic cell death, whereas overexpression of c-FLIP completely averted apoptosis. In addition, high abundance of TRAIL was correlated with poor prognosis in patients with RCC, suggesting that TRAIL, followed by HIF2-alpha and C-FLIP, play a role in the survival and/or progression of malignant RCCs.

\section{INTRODUCTION}

Renal cell carcinoma (RCC) is the urological malignancy with the highest rate of mortality and an increasing incidence worldwide [1]. Clear cell RCC (ccRCC) is the most common form of RCC [2]. Generally, ccRCCs carry genetic insufficiencies of the von HippelLindau tumor suppressor (VHL) and have shown constitutive activation of the hypoxia inducible factors (HIFs). It was previously reported that RCCs expressing HIF2-alpha rather than HIF1-alpha were malignant [3], and that the knockdown of HIF2-alpha induced cell death and/or inhibition of cell growth in RCCs both in vitro and in vivo [3-6].

Previous studies have classified RCC cells into two types, namely starvation-sensitive and starvation-resistant.
Under glucose deprivation, starvation-sensitive RCCs produce $N$-linked (ß-N-acetylglucosamine) ${ }_{2}\left[N\right.$-GlcNAc $\left.{ }_{2}\right]$ modified glycoproteins [7, 8]. These glycoproteins promoted cell death via unfolded protein responses in the endoplasmic reticulum [7, 9]. In contrast, starvationresistant RCCs tolerated the deprivation condition without cytotoxic unfolded protein responses [7], and survived using their enhanced mitochondrial performance and sources of stored carbons [10]. The behavior of the latter type is similar to that of dormant cancer cells, which are often problematic during the therapeutic management of RCC. Interestingly, targeting HIF2-alpha induces cell death in the latter dormant-like RCC [11] (Supplementary Table 1).

This study analyzed the mechanism of cell death caused by inhibition of HIF2-alpha in dormant-like/ 
starvation-resistant RCC. The results demonstrated that cell death was induced by tumor necrosis factor (TNF)related apoptosis-induced ligand (TRAIL) (TNFSF10) [12], and TRAIL-mediated apoptosis was attenuated by HIF2-alpha and c-FLIP. The present study also indicated that higher abundance of TRAIL may be correlated with poor prognosis and has a prognostic value in patients with RCC.

\section{RESULTS}

\section{TRAIL (TNFSF10) is highly expressed in starvation-resistant RCCs}

Global transcriptional data of SW839 versus NC65, which are typical starvation-resistant and starvationsensitive RCC cell lines, respectively, were investigated to clarify the mechanism of cell death induced by inhibition of HIF2-alpha in dormant-like/starvation-resistant RCC. The results showed that TRAIL (TNFSF 10) was highly expressed in SW839 unlike NC65 cells (Figure 1A). TRAIL is a death ligand inducing apoptosis in tumor cells [12]. The expression of TNFSF10 was re-evaluated in three starvation-resistant and four starvation-sensitive RCCs by qRT-PCR analysis (Figure 1B). TNFSF10 was significantly up-regulated in all starvation-resistant RCCs compared with sensitive RCCs. At the protein level, TRAIL up-regulation was generally confirmed in starvation-resistant RCCs, in which cell death was induced by inhibiting HIF2-alpha, compared with those of starvation-sensitive RCCs, with the exception of Cakil (Figure 1C and 1D). These results suggested that TNFSF10 may contribute to the mechanism of cell death caused by inhibiting HIF2-alpha in dormant-like/ starvation-resistant RCC.

\section{HIF2-alpha and c-FLIP avert TRAIL apoptosis in starvation-resistant RCCs}

To clarify the contribution of TNFSF10 in the mechanism of cell death induced by knockdown of HIF2alpha in dormant-like/starvation-resistant RCC, siRNA for TNFSF10 (siTRAIL) was introduced into all three dormant-like/starvation-resistant RCCs, accompanied by siRNA for HIF2-alpha (siHIF2). In the co-introduction of siRNAs for TNFSF10 (siTRAIL) and EPAS1 (siHIF2), siTRAIL signifcantly averted apoptotic cell death induced by siHIF2 in dormant-like/starvation-resistant RCC (Figures 2 and 3). Knockdown of death receptor 5 (DR5), a death receptor for TRAIL [13], via introduction of siDR5 also reduced apoptotic cell death induced by siHIF2. However, silencing of death receptor 4 (DR4), another death receptor for TRAIL [14], did not produce the same result (Figures 2 and 3). Although the efficiencies of DR4 and DR5 knockdowns were similar, the differences between the biological effects of siDR4 and siDR5 may depend on each basal expression of them in starvationresistant cell lines, because the RPKM value of DR5 was approximately 10-fold greater than that of DR4 in SW839 cells (Supplementary Figure 1). Therefore, apoptotic cell death related to TRAIL may be averted by HIF2-alpha in dormant-like/starvation-resistant RCCs. FADD-like apoptosis regulator, c-FLIP $(C F L A R)$ is annotated as an inhibiting gene for TRAIL-induced apoptosis [15]. Expression of c-FLIP was reduced by knockdown of HIF2-alpha in three dormant-like/starvation-resistant RCC cell lines (Figure 4A-4C), whereas knockdown of c-FLIP (siFLIP) induced apoptotic cell death more rapidly and effectively than knockdown of HIF2-alpha in starvationresistant RCC (Figures 4D and 5A). Overexpression of c-FLIP, introduced lentivirally into three dormant-like/ starvation-resistant RCC cell lines, completely cancelled apoptotic cell death induced by siHIF2 (Figures 4E, 5B and Supplementary Figure 2). These data demonstrated that dormant-like/starvation-resistant RCCs evaded TRAIL-induced apoptotic cell death by HIF2-alpha activation followed by induction of c-FLIP.

\section{Abundance of TRAIL (TNFSF10) may be associated with poor prognosis in patients with metastatic RCC}

The role of TRAIL (TNFSF10) expression as a predictor of clinical outcome in patients with metastatic RCC treated with inhibitory agents for tyrosine kinase and/or mTOR in the Cancer Institute Hospital was investigated. The characteristics and demographic data of the 16 patients examined in the cohort are shown in Supplementary Table 2. These patients were classifed into two groups based on the average value of TNFSF 10 expression levels (Figure 6); patients with higher TNFSF10 expression levels had significantly shorter survival periods than those with lower TNFSF10 levels (7.3 vs. 30.1 months, respectively) (Figure 6). This comparative analysis suggested that patients with higher TNFSF 10 expression were resistant to commonly used agents targeting tyrosine kinase and mTOR.

\section{DISCUSSION}

The present study showed that TRAIL-induced apoptosis may be evaded by HIF2-alpha activation followed by induction of c-FLIP in dormant-like/starvationresistant RCC. Cells evaded apoptotic cell death with higher HIF2-alpha levels followed by abundant c-FLIP expression, despite TRAIL being highly expressed in these cells. Subsequently, the knockdown of HIF2-alpha reduced c-FLIP expression and induced apoptotic cell death due to abundant TRAIL. TRAIL-induced apoptotic cell death may be specific to dormant-like/starvation-resistant RCC, which co-expressed HIF2-alpha and TRAIL. Upregulation of c-FLIP has been found in various tumor types, and 
c-FLIP is an important target for cancer therapy using TRAIL [15]. Our immunohistochemical analysis revealed an association of c-FLIP up-regulation with shortly suvival periods in RCC patients (Supplementary Figure 3B-3C). This is the first study showing that the knockdown of HIF2-alpha reduced the expression of c-FLIP. In pancreatic cancer cells with abundant HIF2-alpha, the knockdown of HIF2-alpha induced apoptotic cell death via endogenous TRAIL, regulated by Survivin rather than c-FLIP [16]. Malignat cells driven by high levels of HIF2-alpha can evade apoptotic cell death triggered by TRAIL, with
c-FLIP following sustainable HIF2-alpha. This evidence suggests that HIF2-alpha may be a potentially new therapeutic target for TRAIL-resistant cancer cells.

TRAIL was highly expressed in starvation-resistant RCC cells compared with starvation-sensitive RCC cells. Expression of TRAIL may be promoted by FOXO following the down-regulation of the PI3K-Akt signaling pathway. However, further investigation is warranted to confirm this hypothesis. Global transcriptional data of SW839, a typical starvation-resistant RCC cell line, indicated that "Class I PI3K signaling events mediated
A

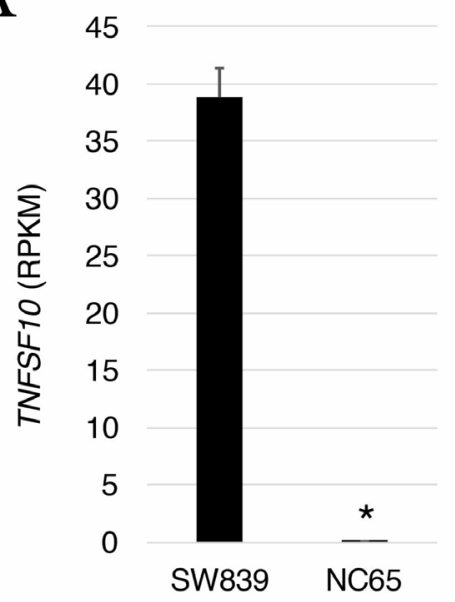

$\mathrm{B}$

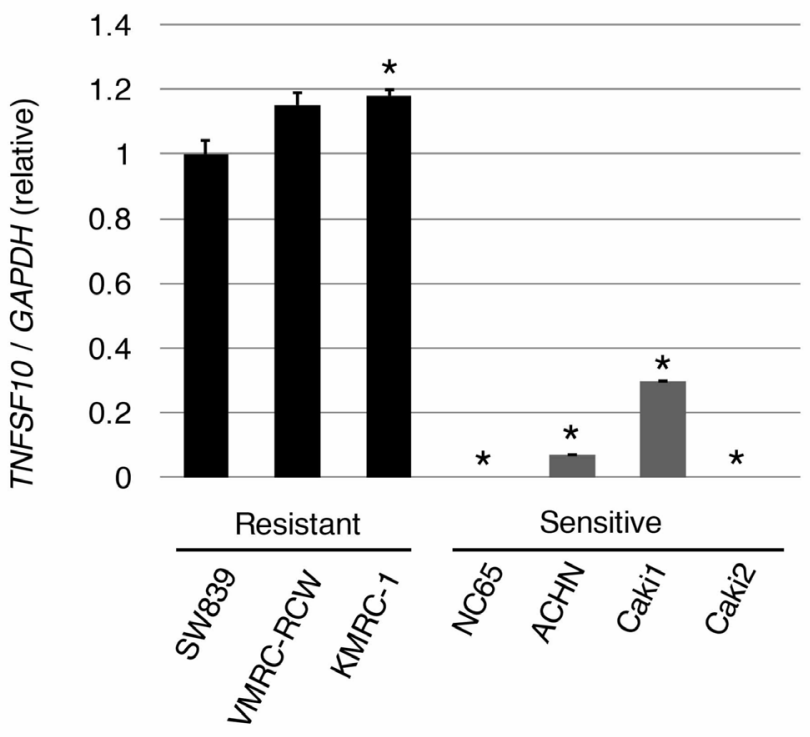

$\mathrm{C}$

TRAIL
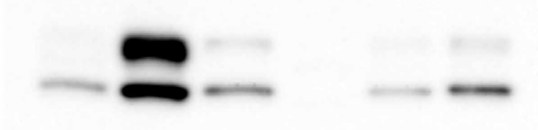

$\alpha$-Tubulin

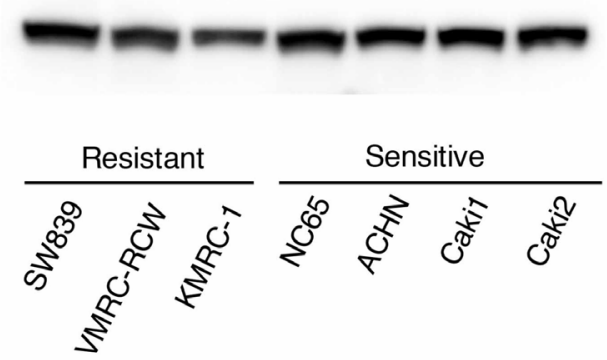

$\mathrm{D}$

- $30 \mathrm{kd}$ 争 $28 \mathrm{kd}$

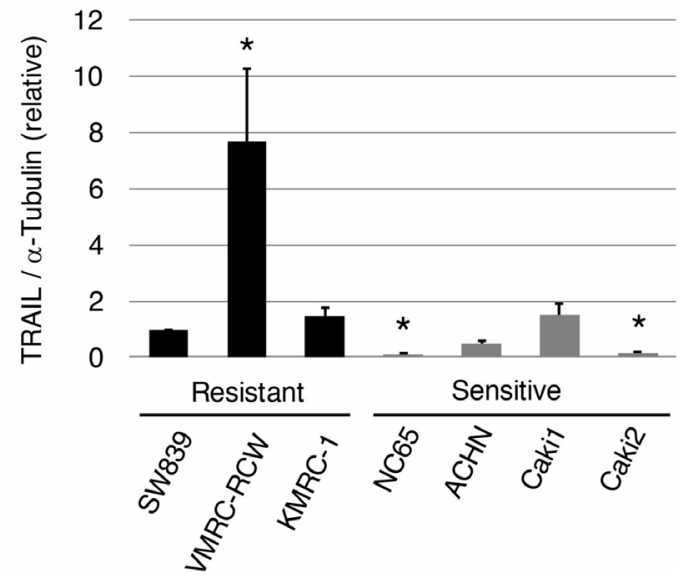

Figure 1: Abundant expression of TRAIL in starvation-ressistant renal cell carcinoma. (A) RPKM value of TNFSF10 (TRAIL) in the starvation-resistant SW839 RCC cell line and the starvation-sensitive NC65 RCC cell line. (B) Quantitative RT-PCR of TRAIL in the starvation-resistant (SW839, VMRC-RCW, and KMRC-1) and starvation-sensitive (NC65, ACHN, Caki1, and Caki2) RCC cell lines. (C) Data shown in panel B for TRAIL were confirmed by western blotting. (D) Quantitative graphic representation of data shown in panel C. Transcriptional and protein expressions were normalized against GAPDH and $\alpha$-tubulin, respectively. Error bars represent standard errors from three independent experiments. ANOVA: $\mathrm{F}(6,14)=336.47, p=2.614 \mathrm{e}^{-14} ; p<0.05$, pairwise comparisons using $t$ tests with pooled SD vs. SW839 $\left(^{*}\right)$. Note that TRAIL mRNA and protein expression was higher in starvation-resistant cells than in starvation-sensitive cells. 


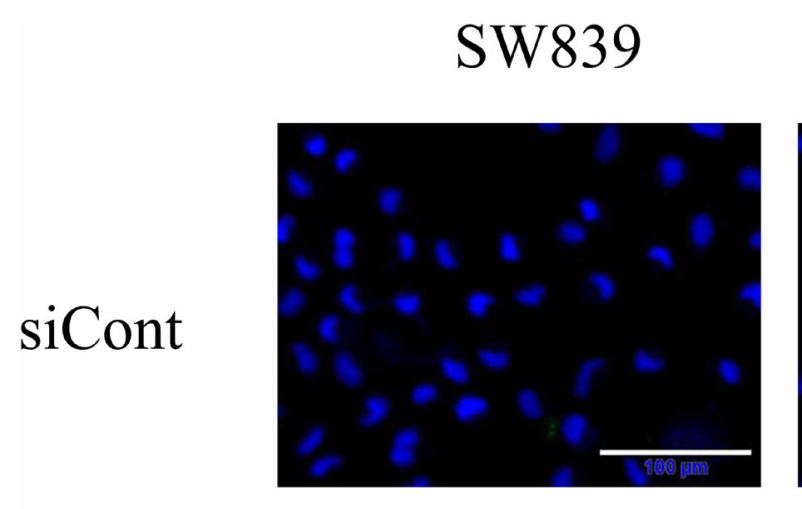

KMRC-1
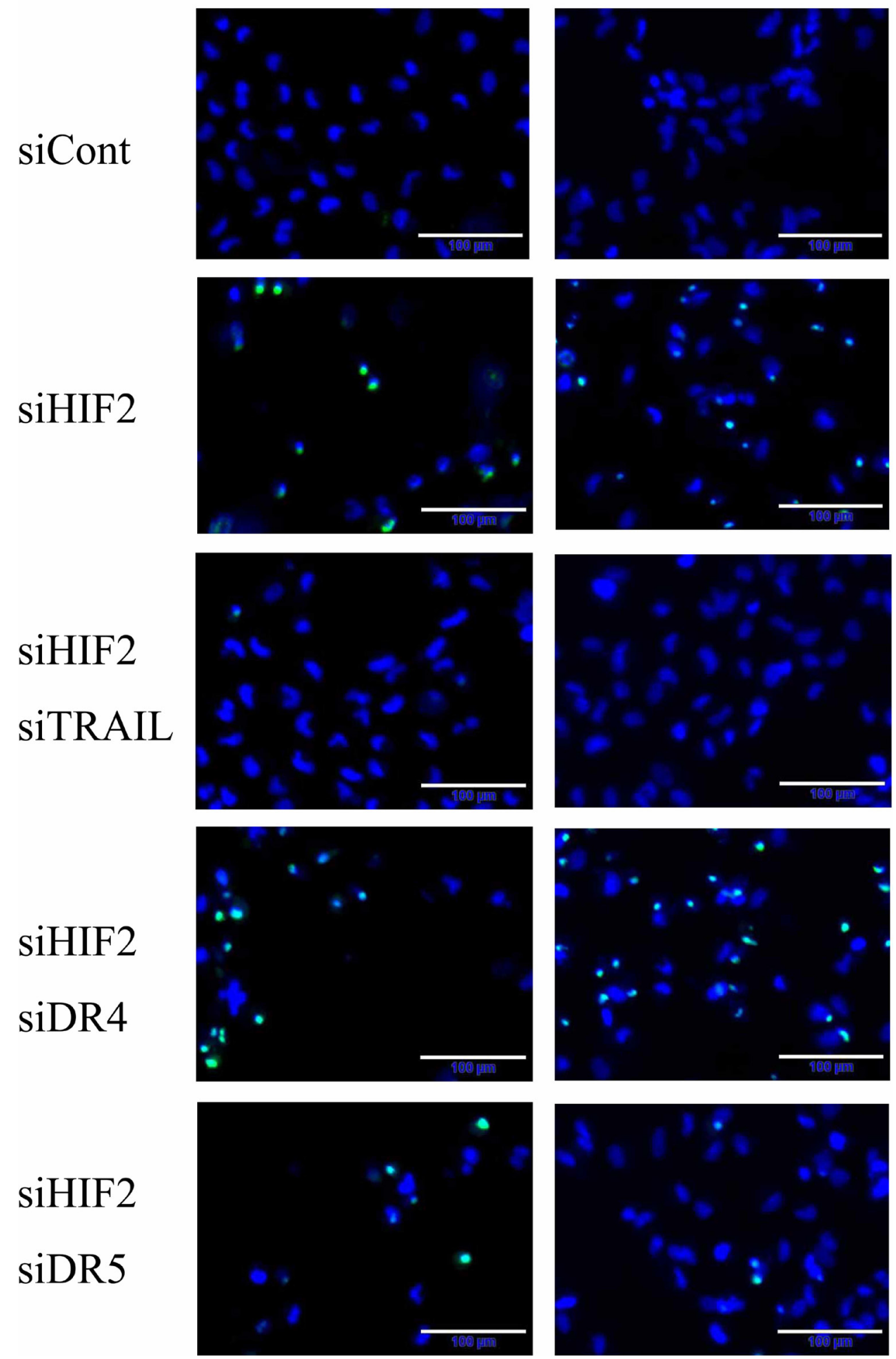

Figure 2: Analysis of apoptosis signals in starvation-resistant RCC. Photographs of signals of CellEvent ${ }^{\mathrm{TM}}$ Caspase-3/7 Green Detection Reagent (apoptotic cells, green) and Hoechet33342 (nuclei of total cells, blue) in the starvation-resistant cells SW839 and KMRC-1 after 2-day treatment with the indicated siRNA reagents. Note that the apoptotic cell death induced by knockdown of HIF2-alpha (siHIF2) in starvation-resistant RCCs was averted by knockdowns of TRAIL (siTRAIL) and its receptor DR5 (siDR5). 
by Akt" was down-regulated, in contrast to the data of NC65, which was a typical starvation-sensitive cell [11]. The PI3K-Akt signaling can inhibit transcriptional activity of FOXO, and TRAIL is a target of "FOXO signaling pathway", according to the database of Kyoto Encyclopedia of Genes and Genomes (KEGG). Inhibition of PI3K-Akt signaling may actively promote FOXO transcription followed by high expression of TRAIL in starvation-resistant RCC cells.

RCCs expressing high levels of TRAIL were associated with poor prognosis. Therefore, expression levels of TRAIL may become a predictive biomarker for the prognosis of clinical outcome in patients with metastatic RCCs. The present investigation was a pilot study involving a limited number of patients treated by various agents. Further clinical studies are warranted to confirm the prognostic value of TRAIL abundance. The findings presented herein were surprising, considering that TRAIL is essentially a ligand associated with apoptosis in cancer cells. The mode of action of abundant TRAIL in dormant-like/starvation-resistant RCCs remains unclear. TRAIL was detected in exosomes prepared from dormant-like/starvation-resistant RCCs (Supplementary Figure 3A). Exosomes were recently reported to contribute to progression of certain types of cancers [17]. Thus, TRAIL-bearing exosomes may contribute to the progression of disease. In the present immunohistochemical analysis (Supplementary Figure 3B), TRAIL was detected with infiltrating lymphocytes in all the tumors, although tumorous TRAIL was found in only 1 out of 30 cases of RCCs. Exosomal TRAIL was hardly detected using immunohistochemistry. Abundance of c-FLIP/CFALR in the tumor was found predominantly in the cases with short survival (Supplementary Figure $3 \mathrm{~B}$, lower row) rather than in the cases with longer survival (Supplementary Figure 3B, upper row), and this

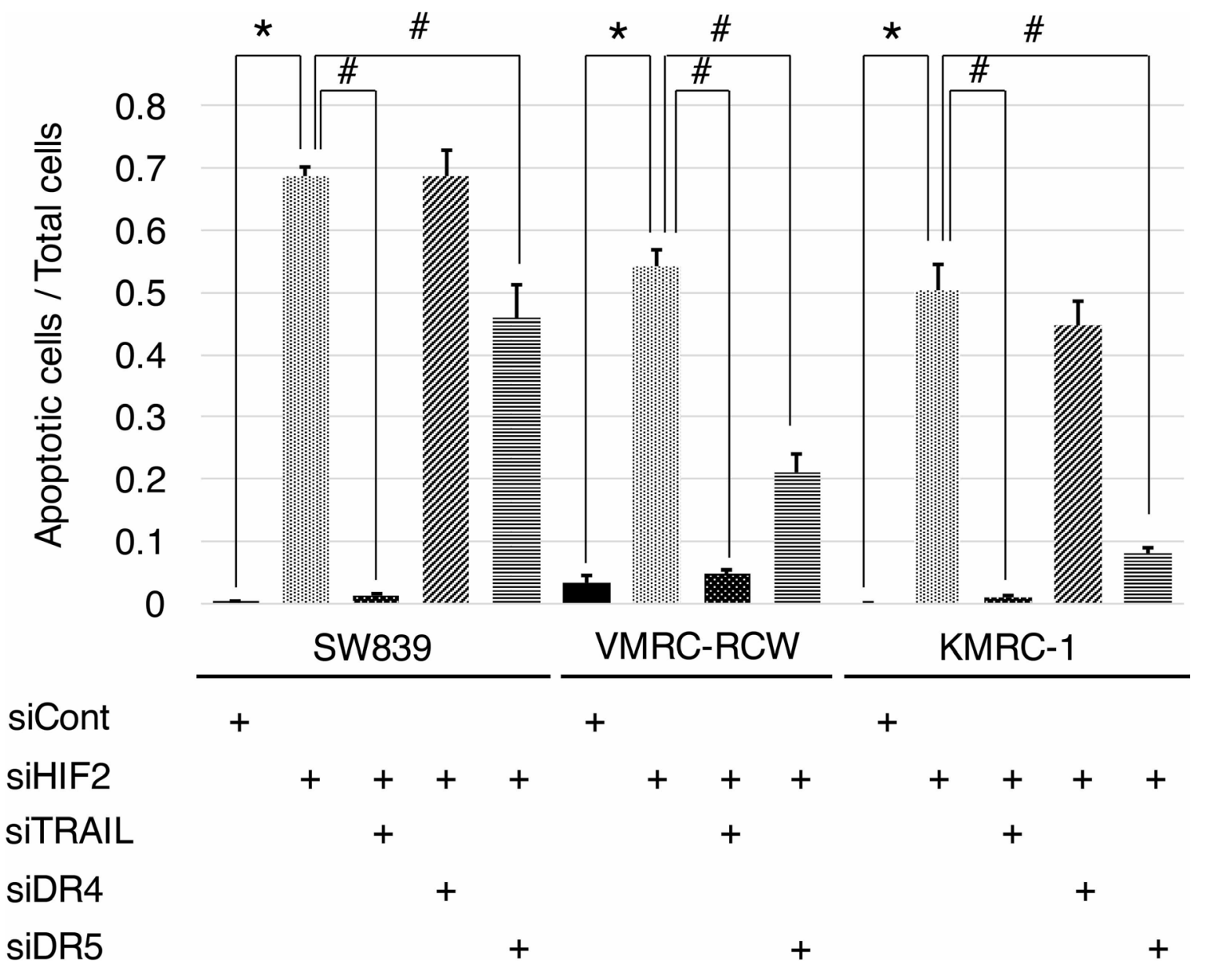

Figure 3: Graphic representation of apoptosis signals in starvation-resistant RCC cell lines. Signals of CellEvent ${ }^{\mathrm{TM}}$ Caspase-3/7 Green Detection Reagent (apoptotic cells, green) and Hoechet33342 (nuclei of total cells, blue) shown in Figure 2 were quantified in all starvation-resistant RCC cell lines (SW839, VMRC-RCW, and KMRC-1). Error bars represent standard errors from six independent experiments. ANOVA: In SW839, F $(4,25)=127.76, p=2.955 \mathrm{e}^{-16}$; in VMRC-RCW, F $(3,20)=120.46, p=5.675 \mathrm{e}^{-13}$; in KMRC-1, $\mathrm{F}(4,25)=89.997, p=1.818 \mathrm{e}^{-14} ; p<0.05$, pairwise comparisons using $t$ tests with pooled SD vs. siCont $\left({ }^{*}\right)$ or siHIF2 $\left({ }^{*}\right)$, respectively. Note that apoptotic cell death induced by knockdown of HIF2-alpha (siHIF2) in starvation-resistant RCCs was signifcantly averted by knockdowns of TRAIL (siTRAIL) and its receptor DR5 (siDR5). 


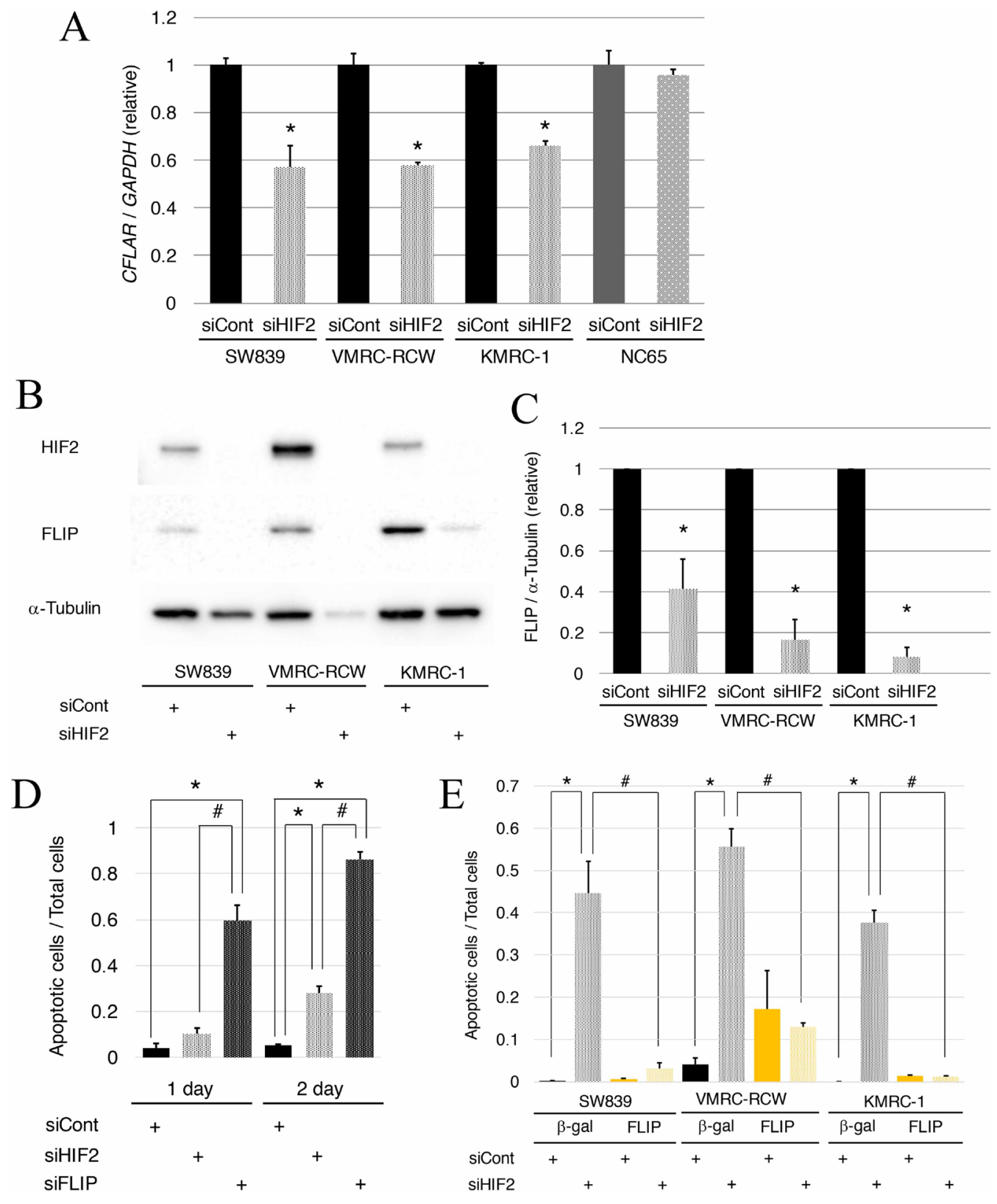

Figure 4: Reduction of c-FLIP following HIF2-alpha knockdown affects cell survival in starvation-resistant RCCs. (A) Quantitative RT-PCR of c-FLIP (CFLAR) in starvation-resistant RCC cell lines (SW839, VMRC-RCW, and KMRC-1) and the starvation-sensitive cell line (NC65) after 2-day treatment with siHIF2. (B) Immunoblots of c-FLIP in all starvation-resistant RCC cells after 2-day treatment with siHIF2. (C) Graphic representation of data shown in panel B. Transcriptional (A) and protein expressions (B-C) were normalized against GAPDH and $\alpha$-tubulin, respectively. Error bars represent standard errors from three independent experiments. Student's $t$-test (two-tailed) was used to compare with each control (siCont). Asterisks indicate $p<0.05$. (D) Quantified graph of signals of CellEvent ${ }^{\mathrm{TM}}$ Caspase-3/7 Green Detection Reagent (apoptotic cells, green) and Hoechet33342 (nuclei of total cells, blue) in the starvationresistant SW839 RCC cell line under 1-day and 2-day treatments with the indicated siRNA reagents (siCont, siHIF2, and siFLIP). Error bars represent standard errors from six independent experiments. ANOVA: 1-day, $\mathrm{F}(2,15)=49.388, p=2.514 \mathrm{e}^{-7} ; 2$-day, $\mathrm{F}(2,15)=290.18$, $p=1.023 \mathrm{e}^{-12} ; p<0.05$, pairwise comparisons using $t$ tests with pooled SD vs. siCont ( $\left.{ }^{*}\right)$ or siHIF2 ( ${ }^{*}$ ), respectively. (E) Quantified graph of signals of CellEvent ${ }^{\mathrm{TM}}$ Caspase-3/7 Green Detection Reagent (apoptotic cells, green) and Hoechet33342 (nuclei of total cells, blue) in all starvation-resistant RCC cell lines, in which $\beta$-gal or c-FLIP was introduced lentivirally, after 2-day treatment with siHIF2. Error bars represent standard errors from five or six independent experiments. ANOVA: In SW839, F $(3,20)=33.782, p=5.047 \mathrm{e}^{-8}$; in VMRC$\mathrm{RCW}, \mathrm{F}(3,20)=20.269, p=2.809 \mathrm{e}^{-6}$; in KMRC-1, $\mathrm{F}(3,19)=155.57, p=1.506 \mathrm{e}^{-13} ; p<0.05$, pairwise comparisons using $t$ tests with pooled SD vs. siCont $\left(^{*}\right)$ or siHIF2 $(*)$, respectively, of the control cells, in which $\beta$-gal was introduced lentivirally. Note that knockdown of c-FLIP induced augmentation of apoptosis with knockdown of HIF2-alpha (D), and that artificial introduction of c-FLIP averted apoptosis completely in all starvation-resistant RCC cell lines (E). 
A

siCont

siFLIP

B

siCont

siHIF2 1day
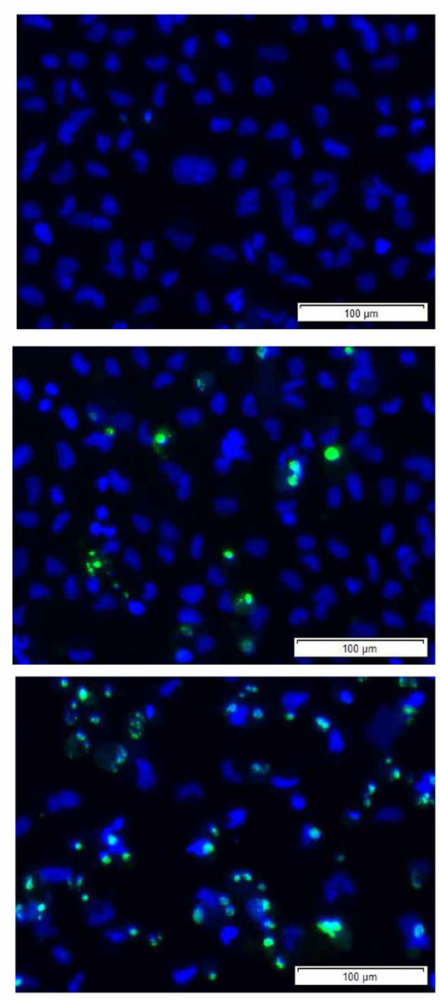

$\beta$-gal
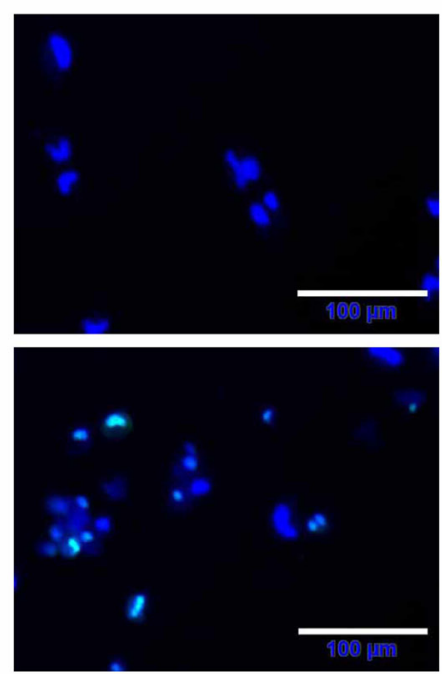

2day
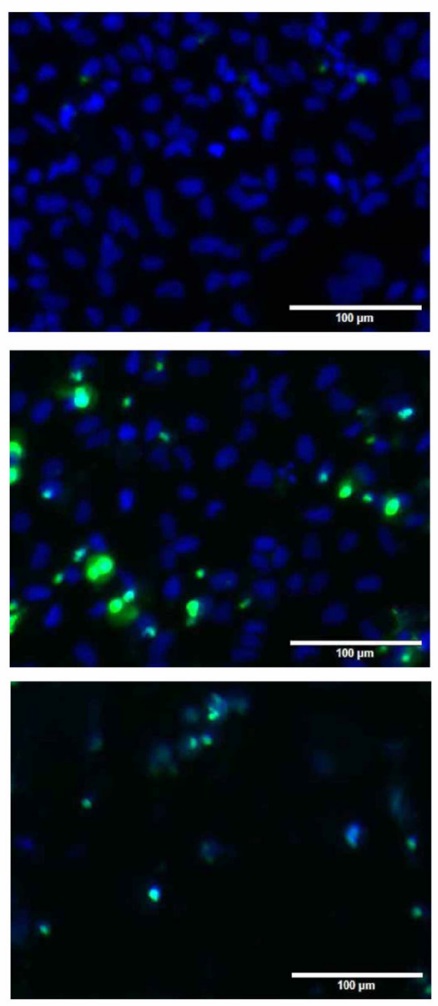

FLIP
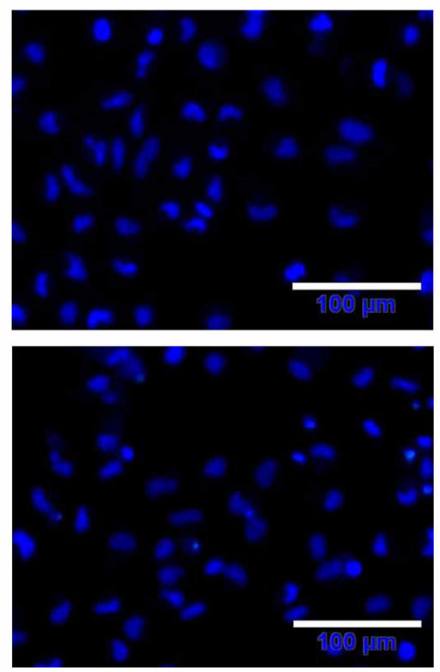

Figure 5: Knockdown of c-FLIP induces apoptosis and its introduction averts apoptotic cell death in the starvationresistant RCC cell line SW839. (A) Apoptotic signals of CellEvent ${ }^{\mathrm{TM}}$ Caspase-3/7 Green Detection Reagent (apoptotic cells, green) and Hoechet33342 (nuclei of total cells, blue) in the starvation-resistant RCC cell line SW839 under 1-day and 2-day treatments with the indicated siRNA reagents (siCont, siHIF2 and siFLIP). (B) Photographic representation of SW839 cells, in which $\beta$-gal or c-FLIP was introduced lentivirally, after 2-day treatment with siHIF2. Note that knockdown of c-FLIP induced similar or greater apoptosis, and exogeneous introduction of c-FLIP completely averted apoptosis with siHIF2 in SW839 cells. These are photographic representations of data shown in Figure 4D and 4E. 
difference was statistically significant (Supplementary Figure 3C). Therefore, these data suggested that RCCs with poor prognosis may evate TRAIL-induced apoptosis through high expression of c-FLIP, and this may be due to sustainable HIF2-alpha expression.

In conclusion, TRAIL-induce apoptosis was attenuated by c-FLIP following sustainable HIF2-alpha expression in dormant-like/starvation-resistant RCC. Abundance of TRAIL may foster the development of RCCs, and affect their malignancy and prognosis in RCCs (Figure 7).

\section{MATERIALS AND METHODS}

\section{Cell lines and cell culture conditions}

Three starvation-resistant RCC cell lines (SW839, VMRC-RCW and KMRC-1) and four starvation-resistant RCC cell lines (Caki1, Caki2, NC65 and ACHN) were used in this study. These cell lines were purchased from either the American Type Culture Collection, Riken Cell Bank, Cell Resource Center for Biomedical Research in Tohoku University (Sendai, Japan) or the Japanese Collection of Research Bioresources (Osaka, Japan). All cell lines were maintained in RPMI 1640 (Nakarai Tesque, Kyoto, Japan), containing $25 \mathrm{mM}$ glucose, supplemented with $10 \%$ fetal calf serum, penicillin $(100 \mathrm{U} / \mathrm{ml})$ and streptomycin $(100$ $\mu \mathrm{g} / \mathrm{ml})$ at $37^{\circ} \mathrm{C}$ in a humidified $5 \% \mathrm{CO}_{2}$ atmosphere.

\section{Patients}

The medical records of patients with $\mathrm{RCC}$, treated in the Cancer Institute Hospital (Japanese Foundation for Cancer Research, Tokyo, Japan) between 2008 and 2014, were retrospectively reviewed. In all patients, ccRCC was confirmed by pathological diagnosis. Histopathology was reviewed according to the 2004 World Health Organization classification [18]. This study was carried out in compliance with the Helsinki declaration, and was approved by the institutional review board of the Cancer Institute Hospital (2009-1029). Written informed consent was obtained from all patients in this study.

\section{Antibodies}

The anti-TRAIL (\#3219) and anti-HIF2- $\alpha$ (\#7096) rabbit monoclonal antibodies were purchased from Cell Signaling Technology (Beverly, MA, USA). The anti-CD63 (ab134045) rabbit monoclonal antibody was purchased from Abcam (Cambridge, MA, USA). The anti-c-FLIP (sc-5276) mouse monoclonal antibody was purchased from Santa Cruz Biotechnology (Santa Cruz, CA, USA). The anti- $\alpha-$
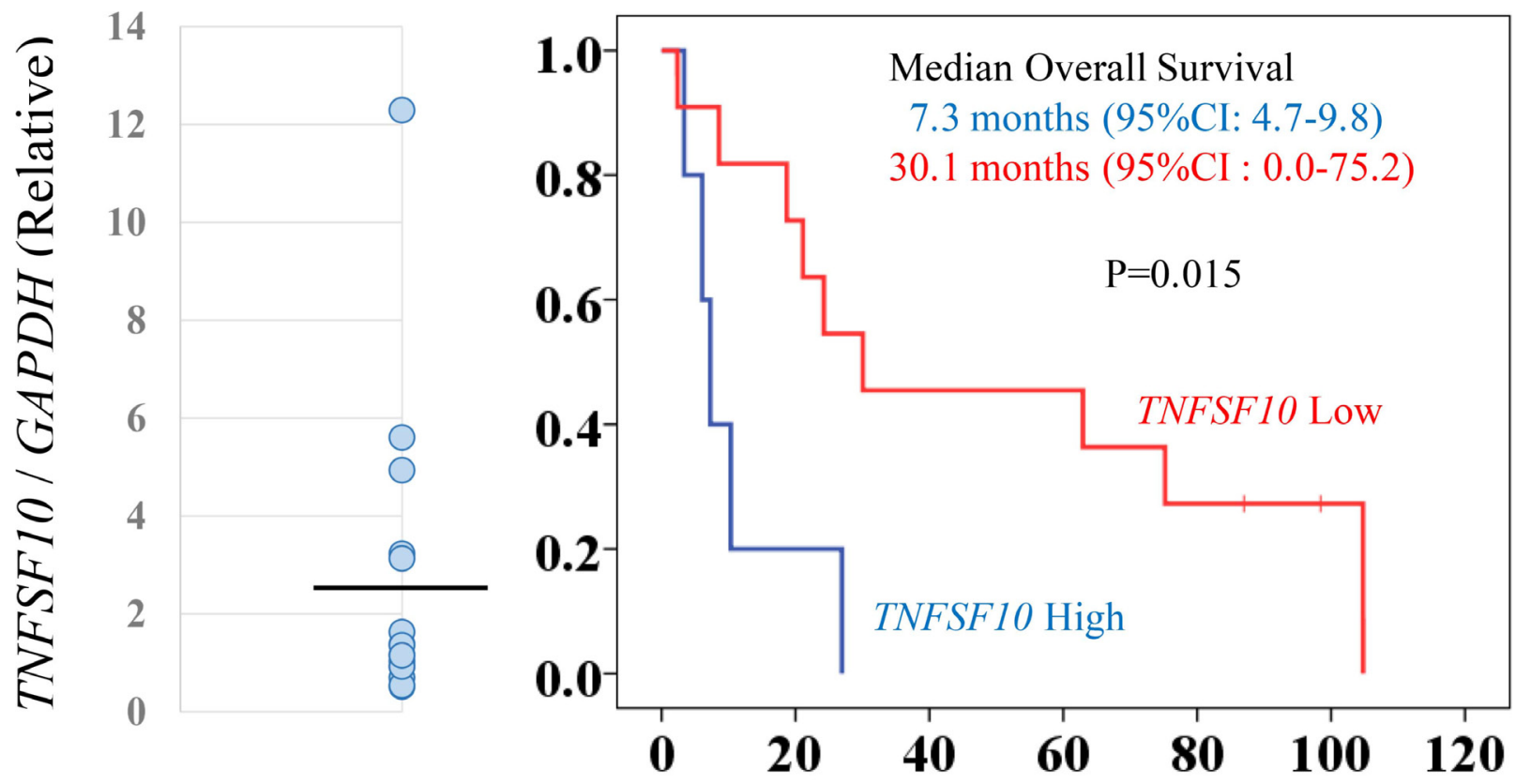

\section{Month}

Figure 6: TNFSF10 (TRAIL) expression levels associated with prognosis for patients with metastatic RCC. To determine TNFSF10 (TRAIL) expression levels, clinical samples from patients with metastatic RCCs were analyzed by qRT-PCR with GAPDH normalization (left panel). The black line indicates the average value of TNFSF10 expression. The RCC patients were categorized in high or low TNFSF10 expression groups based on the average value. Kaplan-Meier survival curves for TNFSF10 in patients with metastatic RCC (right panel). The high TNFSF10 group was associated with significantly shorter survival periods compared with the low TNFSF10 group after chemotherapy (log-rank test, $p=0.015$ ). 
tubulin (\#T9026, DM1A) mouse monoclonal antibody was purchased from Sigma-Aldrich (St. Louis, MO, USA).

\section{Immunoblotting}

Cells were lysed in Laemmli-SDS buffer, subjected to SDS-polyacrylamide gel electrophoresis, and electrotransferred to membrane filters (Immuno-Blot ${ }^{\circledR}$ PVDF membranes, Bio-Rad Laboratories, Richmond, CA, USA). The filters were incubated overnight with a primary antibody in TBS-T buffer containing $2 \%$ bovine serum albumin (BSA) and incubated for 1 hour in horseradish peroxidase-conjugated anti-mouse or anti-rabbit secondary antibody (Cell Signaling Technology) diluted 1:5,000 in TBS-T buffer containing 2\% BSA. Immunoreactivity was detected using the Luminata ${ }^{\mathrm{TM}}$ Classico Western HRP substrate (Millipore Corporation, Billerice, MA, USA) with LAS4000 (Fujifilm, Tokyo, Japan) and quantified using the MultiGauge ${ }^{\mathrm{TM}}$ software (Fujifilm) against anti$\alpha$-tubulin antibody as internal control.

\section{Analysis of apoptosis signals}

Cells $\left(1 \times 10^{4}\right) / 100 \mu$ were plated onto 96-well culture plates and cultured at $37^{\circ} \mathrm{C}$. After treatment, $5 \mu \mathrm{M}$ CellEvent ${ }^{\mathrm{TM}}$ Caspase-3/7 Green Detection Reagent (Thermo Fisher Scientific, Waltham, MA, USA) and $10 \mu \mathrm{g} / \mathrm{ml}$ Hoechet33342 (Dojindo, Mashiki, Kumamoto, Japan) were added to the treated cells and the plates were incubated for 30 minutes at room temperature. These cells were observed using a fluorescence microscope IX83 (Olympus, Tokyo, Japan) and analyzed using the imaging software cellSens (Olympus).

\section{Quantitative reverse transcription-polymerase chain reaction (qRT-PCR)}

Total RNA was obtained from RCC cells and tissues of patients who had undergone radical nephrectomy using acid guanidinium thiocyanatephenol-chloroform [19]. Quantitative RT-PCR was performed using the LightCycler ${ }^{\circledR} 480$ SYBG Master I Mix and LightCycler ${ }^{\circledR} 480$ System II (Roche Diagnostics, Mannheim, Germany). Gene expression was normalized against the GAPDH gene. Primer sequences are listed in Supplementary Table 3A. All quantification analyses were performed in triplicate.

\section{SiRNA}

RNA duplexes for siRNAs targeting human HIF2alpha (EPAS1, s4699), TRAIL (TNFSF10, s16664), c-FLIP (CFLAR, s16864), DR4 (TNFRSF10A, s16764) and DR5 (TNFRSF10B, s16756) were purchased from Life Technologies. Scrambled control RNA duplexes (Silencer ${ }^{\circledR}$ Select Negative Control \#1 siRNA, 4390844) were also purchased from Life Technologies. Cells were transfected with RNA duplexes using Lipofectamine ${ }^{\circledR}$ RNAiMAX reagents (Thermo Fisher Scientific) according to the manufacturer's protocol.

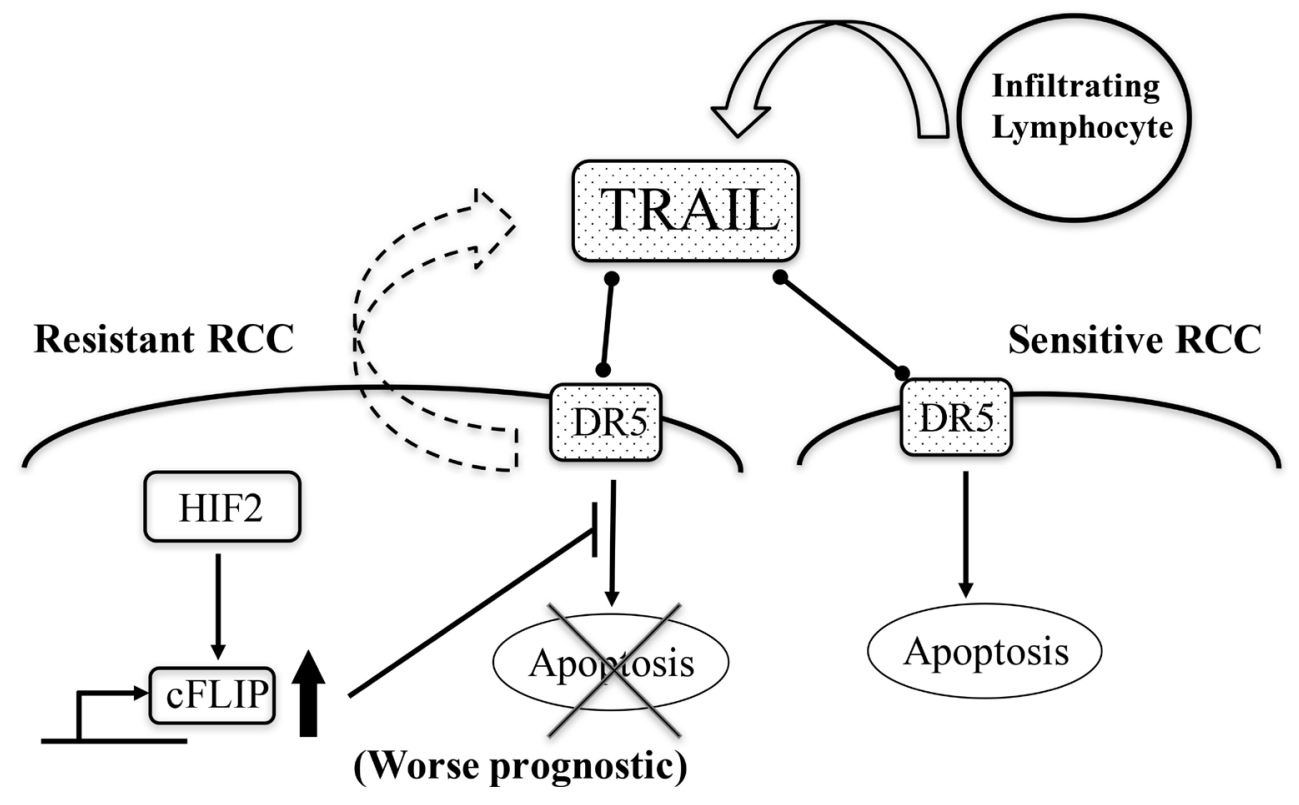

Figure 7: A schematic summary of all experimental results. TRAIL abundance caused by infiltraring lymphocytes and exosomes from starvation-resistant RCC cells induce apoptosis into starvation-sensitive RCC cells; however, the dormant-like/resistant RCCs may evade TRAIL-induced apoptosis, with c-FLIP following sustainable expression of HIF2-alpha. Subsequently, abundance of TRAIL may foster the development of RCCs and affect malignancy and prognosis of RCCs. Thin arrows indicate activation of a target and events. The thick arrow pointing upwards signifies an increase in the levels of the corresponding molecules. The cross signifies inhibition of an event. The bar arrow indicates suppression of a target. Dot arrows indicate molecular interactions. Curved arrows indicate secretion of vesicles. 


\section{Preparation of lentiviruses}

Lentiviral cDNA vectors for CFLAR were obtained from Dharmacon (GE Dharmacon, Cambridge, UK). pLenti6 vector containing $\beta$-gal cDNA (Invitrogen, Thermo, MA, USA) was used as overexpression control. Lentiviruses transferring cDNA were prepared using the Lenti- $\mathrm{X}^{\mathrm{TM}}$ HTX packaging system (Clontech, Takara Bio, Shiga, Japan) according to manufacturer's instructions. SW839, VMRC-RCW and KMRC-1 cells transferred by $>20$ MOI of each lentivirus were selected in the presence of $10 \mu \mathrm{g} / \mathrm{ml}$ blasticidin, expanded and used in the experiments.

\section{Analysis of global transcriptional data}

Global transcriptional data for starvation-resistant RCC cell line SW839 and for starvation-sensitive RCC cell line NC65 were acquired from the DDBJ under accession number DRA005074, PRJDB5127, and SAMD0005806873 as described previously [11].

\section{Statistics}

The data are reported as means \pm standard error (SE). The values were derived from at least three replicate experiments. Statistical analyses were performed using the free software R. One-way factorial analysis of variance (ANOVA), accompanied by pairwise comparisons using $t$ tests with pooled standard deviation (SD) was used to compare multiple group means. Student's $t$-test (two-tail) was used to compare the means of two groups. A $p$-value of $<0.05$ was considered to denote statistical significance. In the clinical study, overall survival periods were measured from the day of initial administration of the therapeutic agents until the day of disease-specific death. Time-to-event distributions were estimated using Kaplan-Meier curves and statistically analyzed (log-rank test) using the SPSS software (SPSS for Windows, version 17.0, SPSS Inc.).

\section{Author contributions}

T. I. designed the study, performed experiments, analyzed data and wrote the article. T.C. performed experiments and assisted in the preparation of the article. T. Y., S. K., A. K., and J. Y. provided clinical materials and data. T. Y. analyzed the clinical data and assisted in the preparation of the article.

\section{ACKNOWLEDGMENTS}

The authors thank Mr. Shouhei Ishida, Mr. Takefumi Yamamoto and Ms. Hiroko Kita (Central Reseach Laboratory, Shiga University of Medical Science) for their assistance with optical observations and qPCR analysis.

\section{CONFLICTS OF INTEREST}

The authors have no conflicts of interest to declare.

\section{FUNDING}

This work was supported by the Smoking Research Foundation (T. Y.); JSPS KAKENHI Grant Numbers 15K06854 (T. I.), 18K09192 (T. C. and S. K.), and 16K11035 (T. Y.).

\section{REFERENCES}

1. Siegel RL, Miller KD, Jemal A. Cancer statistics, 2015. CA Cancer J Clin. 2015; 65:5-29.

2. Keith B, Johnson RS, Simon MC. HIF1a and HIF2a: sibling rivairy in hypoxic tumour grouth and progression. Nat Rev Cancer. 2011; 15:9-22.

3. Koh MY, Nguyen V, Lemos R Jr, Darnay BG, Kiriakova G, Abdelmelek M, Ho TH, Karam J, Monzon FA, Jonasch E, Powis G. Hypoxia-induced SUMOylation of E3 ligase HAF determines specific activation of HIF2 in clear-cell renal cell carcinoma. Cancer Res. 2015; 75:316-329.

4. Qiu B, Ackerman D, Sanchez DJ, Li B, Ochocki JD, Grazioli A, Bobrovnikova-Marjon E, Diehl JA, Keith B, Simon MC. HIF2 $\alpha$-Dependent Lipid Storage Promotes Endoplasmic Reticulum Homeostasis in Clear-Cell Renal Cell Carcinoma. Cancer Discov. 2015; 5:652-667.

5. Kondo K, Kim WY, Lechpammer M, Kaelin WG Jr. Inhibition of HIF2alpha is sufficient to suppress pVHL-defective tumor growth. PLoS Biol. 2003; 1:439-444.

6. Zimmer M, Doucette D, Siddiqui N, Iliopoulos O. Inhibition of hypoxia-inducible factor is sufficient for growth suppression of VHL-/- tumors. Mol Cancer Res. 2004; 2:89-95.

7. Isono T, Chano T, Kitamura A, Yuasa T. Glucose deprivation induces $\mathrm{G} 2 / \mathrm{M}$ transition-arrest and cell death in N-GlcNAc2-modified protein-producing renal carcinoma cells. PLoS One. 2014; 9:e96168.

8. Isono T. O-GlcNAc-specific antibody CTD110.6 cross-reacts with N-GlcNAc2-modified proteins induced under glucose deprivation. PLoS One. 2011; 6:e18959.

9. Isono T, Chano T, Okabe H, Suzaki M. Study of global transcriptional changes of N-GlcNAc2 proteins-producing T24 bladder carcinoma cells under glucose deprivation. PLoS One. 2013; 8:e60397.

10. Isono T, Chano T, Yonese J, Yuasa T. Therapeutic inhibition of mitochondrial function induces cell death in starvation-resistant renal cell carcinomas. Sci Rep. 2016; 6:e25669.

11. Isono T, Chano T, Yoshida T, Kageyama S, Kawauchi A, Suzaki M, Yuasa T. Hydroxyl-HIF2-alpha is potential therapeutic target for renal cell carcinomas. Am J Cancer Res. 2016; 6:2263-2276. 
12. Wiley SR, Schooley K, Smolak PJ, Din WS, Huang CP, Nicholl JK, Sutherland GR, Smith TD, Rauch C, Smith CA, Goodwin RG. Identification and characterization of new member of the TNF family that induced apoptosis. Immunity. 1995; 3:673-682.

13. Walczak H, Degli-Esposti MA, Jhonson RS, Smolak PJ, Waugh JY, Boiani N, Timour MS, Gerhart MJ, Schooley KA, Smith CA, Goodwin RG, Rauch C. TRAIL-R2: a novel apoptosis-mediating receptor for TRAIL. EMBO J. 1997; 16:5386-5397.

14. Pan G, O'Rourke K, Chinnaiyan AM, Gentz R, Ebner R, Ni J, Dixit VM. The receptor for the cytotoxic ligand TRAIL. Science. 1997; 276:111-113.

15. Safa AR, Pollok KE. Targetig the anti-apoptotic Protein c-FLIP for Cancer Therapy. Cancers. 2011; 3:1639-1671.
16. Harashima N, Takenaga K, Akimoto M, Harada M. HIF-2 $\alpha$ dictates the susceptibility of pancreatic cncer cells to TRAIL by regulating survivin expression. Oncotarget. 2017; 8:42887-42900. https://doi.org/10.18632/oncotarget.17157.

17. Ruivo CF, Adem B, Silva M, Melo S. The biology of cancer exosomes: Insights and new perspectives. Cancer Res. 2017; 77:6480-6488.

18. Lopez-Beltran A, Scarpelli M, Montironi R, Kirkali Z. 2004 WHO Classification of the Renal Tumors of the Adults. Eur Urol. 2006; 49:798-805.

19. Chomczynski P, Sacchi N. Single-step method of RNA isolation by acid guanidinium thiocyanate-phenol-chloroform extraction. Anal Biochem. 1987; 162:156-159. 